\title{
Original Article \\ Evaluation of Contraindicated Drug-Drug Interactions in Electronic Health Insurance Data at 3 Hospitals in Quang Ninh Province through Navicat Software
}

\author{
Nguyen Thanh Hai ${ }^{1, *}$, Phung Thi Thu $\mathrm{Ha}^{2}$, Do Thanh Long ${ }^{1}$, \\ Duong Thi Van ${ }^{1}$, Nguyen Xuan Bach ${ }^{3}$ \\ ${ }^{1}$ Hanoi University of Pharmacy, 13-15 Le Thanh Tong, Hoan Kiem, Hanoi, Vietnam \\ ${ }^{2}$ Social insurance at Quang Ninh Province, Tran Hung Dao, Ha Long, Quang Ninh, Vietnam \\ ${ }^{3}$ VNU University of Medicine and Pharmacy, 144 Xuan Thuy, Cau Giay, Hanoi, Vietnam \\ Received 16 October 2020 \\ Revised 25 January 2021; Accepted 25 January 2021
}

\begin{abstract}
Abtract: This study analyzes contraindicated drug-drug interactions stored in electronic health insurance data at three hospitals in Quang Ninh Province based on the list of concentrated contractor drugs applied in Quang Ninh province within the scope of payment of the Health Insurance Fund in 2018. The researchers developed a list of 112 pairs of the contraindicated drug-drug interactions to serve as a reference to determine the frequency of contraindicated drug-drug interactions over 519,500 medical records and 1,254,099 prescriptions at 3 hospitals in Quang Ninh province. Twenty-six contraindicated drug-drug interactions $(0.006 \%)$ were found over 297 medical records and 789 prescriptions; 5 pairs with the highest frequency are: Amiodarone-Amitriptyline, Amiodarone-Moxifloxacin, Amiodarone-Colchicine, Levodopa-Sulpiride, and AmiodaroneHaloperidol. Medical reccords with contraindicated drug-drug interactions were found in 13 different departments or interdisciplinary departments at all the three hospitals, of which the highest rates were found in Intensive Care Unit and Emergency Departments (24.2\%), Endocrinology Departments (16.2\%) and Cardiology Pediatric Departments (10.4\%). The study also analyzed 26 pairs of common contraindicated drug-drug interactions in clinical practice based on retrospective data on drugs prescribed at the 3 hospitals in Quang Ninh Province.
\end{abstract}

Keywords: Contraindicated drug-drug interactions, hospitals in Quang Ninh province, medication review tools.

\footnotetext{
* Corresponding author.

E-mail address: haint@hup.edu.vn

https://doi.org/10.25073/2588-1132/vnumps.4277
} 


\title{
Phân tích tương tác thuốc chống chỉ định trên dữ liệu điện tử bảo hiểm y tế của một số bệnh viện tỉnh Quảng Ninh thông qua phần mềm Navicat ${ }^{\circledR}$
}

\author{
Nguyễn Thành Hải $1^{1, *}$, Phùng Thị Thu Hà ${ }^{2}$ Đỗ Thành Long ${ }^{1}$, \\ Dương Thị Vân ${ }^{1}$, Nguyễn Xuân Bách ${ }^{3}$ \\ ${ }^{1}$ Truoòng Đại học Dược Hà Nội, 13-15 Lê Thánh Tông, Hoàn Kiếm, Hà Nội, Việt Nam \\ ${ }^{2}$ Bảo hiểm Xã hội Tỉnh Quảng Ninh, Trần Hung Đạo, Hạ Long, Quảng Ninh, Việt Nam \\ ${ }^{3}$ Truò̀ng Đại học Y Duoọc, Đại học Quốc gia Hà Nội, Số 144 Xuân Thủy Cầu Giấy, Hà Nội, Việt Nam
}

Nhận ngày 16 tháng 10 năm 2020

Chỉnh sửa ngày 25 tháng 01 năm 2021; Chấp nhận đăng ngày 25 tháng 01 năm 2021

\begin{abstract}
Tóm tắt: Tương tác thuốc (TTT) là vấn đề thường gặp trong thực hành lâm sàng, làm giảm hiệu quả điều trị và có thể dẫn đến các tai biến nguy hiểm cho bệnh nhân, thậm chí dẫn đến tử vong. Mục đích của nghiên cứu này nhằm phân tích các tương tác thuốc chống chỉ định $(\mathrm{CC})$ khi kê đơn tại một số bệnh viện Tỉnh Quảng Ninh. Đối tượng và phương pháp nghiên cứu: danh mục thuốc thầu tập trung được áp dụng trên địa bàn Tỉnh Quảng Ninh và trong phạm vi thanh toán của Quỹ BHYT năm 2018. Kết quả: nhóm nghiên cứu đã xây dựng được danh mục lý thuyết bao gồm 112 cặp TTT CCĐ để làm tham chiếu xác định tần suất xuất hiện TTT CCĐ trên 519500 bệnh án và 1254099 đơn thuốc tại 3 bệnh viện Tỉnh Quảng Ninh. Trong đó, có 26 TTT CCĐ $(0,06 \%)$ được phát hiện trên 297 bệnh án và 789 đơn. 5 cặp TTT CCĐ có tần suất nhiều nhất là: Amiodaron-Amitriptylin, AmiodaronMoxifloxacin, Amiodaron-Colchicin, Levodopa-Sulpirid, Amiodaron-Haloperidon. Bệnh án có TTT CCĐ được phát hiện ở 13 khoa hoặc liên khoa khác nhau từ cả 3 bệnh viện, trong đó tỷ lệ phát hiện cao nhất tại các khoa Hồi sức cấp cứu $(24,2 \%)$, Nội tiết $(16,2 \%)$, Nội tim mạch $(10,4 \%)$ và \%); Lão khoa $(6,1 \%)$. Độ tuổi trung bình của các bệnh nhân có TTT CCĐ ở cả 3 bệnh viện đều lớn hơn hoặc bằng 67 , chủ yếu là bệnh nhân nội trú. Kểt luận: dựa trên hồi cứu dữ liệu lớn về kê đơn thuốc tại 3 bệnh viện thuộc Tỉnh Quảng Ninh, nghiên cứu đã phân tích được 26 cặp TTT CCĐ thường gặp trong thực hành lâm sàng.
\end{abstract}

Tù khóa: Bệnh viện Tỉnh Quảng Ninh, công cụ rà soát kê đơn, tương tác thuốc chống chỉ định.

\section{Mở đầu}

Tương tác thuốc (TTT) bất lợi là một trong những nguyên nhân gây ra các biến cố bất lợi của thuốc, gây giảm hiệu quả điều trị, kéo dài thời gian nằm viện, tăng chi phí điều trị, và thậm chí có thể gây tử vong cho bệnh nhân [1]. Hiện nay, các cơ sở dữ liệu tra cứu TTT rất phong phú, đa dạng bao gồm cả sách chuyên khảo lẫn phần mềm duyệt tương tác trực tuyến. Tuy nhiên, do cơ sở dữ liệu sử dụng trong các phần mềm khác nhau nên kết quả duyệt tương tác có sự khác biệt về mức độ nghiêm trọng và khuyến cáo xử trí [2]. Điều này gây nhiều khó khăn cho bác sỹ,

\footnotetext{
"Tác giả liên hệ.

Địa chi email: haint@hup.edu.vn

https://doi.org/10.25073/2588-1132/vnumps.4277
} 
dược sỹ trong việc chọn lựa nguồn thông tin phù hợp và chính xác. Để khắc phục những khó khăn trên và thuận tiện tra cứu thông tin, nhiều bệnh viện và cơ sở khám chữa bệnh trên thế giới và Việt Nam đã xây dựng bảng danh mục các tương tác thuốc bất lợi riêng trong thực hành lâm sàng [3], sau đó tích hợp vào công cụ rà soát kê đơn điện tử của phần mềm kê đơn tại Bệnh viện nhằm cảnh báo các cặp tương tác nghiêm trọng khi kê đơn. Tỉnh Quảng Ninh là một tỉnh với hơn 300 cơ sở khám, chữa bệnh công lập và ngoài công lập ký hợp đồng Bảo hiểm Y tế (BHYT). Danh mục thuốc được áp dụng lên tới hơn 1000 hoạt chất ( $80 \%$ là thuốc uống). Do đó, TTT đặc biệt các thuốc dạng đường uống là vấn đề không thể tránh khỏi. Nghiên cứu được thực hiện với mục đích phân tích được các cặp TTT CCĐ thường gặp trong thực hành lâm sàng tại 3 bệnh viện Tỉnh Quảng Ninh dựa trên dữ liệu hồi cứu kê đơn lấy từ BHYT Tỉnh.

\section{2. Đối tượng và phương pháp nghiên cứu}

\section{1. Đối tuợng nghiên cứu}

i) Danh mục thuốc thuộc kết quả đấu thầu thuốc tập trung tại Quảng Ninh năm 2018 và trong phạm vi thanh toán của Quỹ BHYT.

- Tiều chuẩn lựa chọn: các hoạt chất nằm trong danh mục thuốc sử dụng trong phạm vi thanh toán của Quỹ BHYT năm 2018 và là thuốc sử dụng theo đường uống.

- Tiêu chuẩn loại trừ: các thuốc: có nguồn gốc từ Dược liệu; chứa Vitamin và khoáng chất; các dạng men và vi khuẩn đông khô.

ii) Dữ liệu các bệnh án điện tử của bệnh nhân tại 3 bệnh viện Tỉnh Quảng Ninh (ký hiệu là Bệnh viện $A$; Bệnh viện $B$ và Bệnh viện $C$ ) trong năm 2018; đây là 3 bệnh viện hạng 1 , quy mô giường bệnh tương đương nhau và là bệnh viện đa khoa.

- Tiêu chuẩn lựa chọn: toàn bộ dữ liệu từ bệnh án điện tử trong khám, chữa bệnh BHYT nội, ngoại trú của 3 Bệnh viện Tỉnh Quảng Ninh (theo dữ liệu trên phần mềm Giám định BHYT).
- Tiêu chuẩn loại trừ: đơn kê sử dụng nhỏ hơn 2 thuốc.

\subsection{Phương pháp nghiên cứu}

i) Xây dựng danh mục tương tác thuốc chống chỉ định bao gồm 5 bước: Bước 1 : xây dựng danh mục hoạt chất để tra cứu trong Micromedex 2.0 (MM 2.0); Bước 2: xây dựng danh mục TTT $\mathrm{CCĐ}$ và nghiêm trọng từ $\mathrm{MM} 2.0$; Bước 3 : xây dựng danh mục TTT CCĐ thông qua sự dồng thuận trên các khuyến cáo từ tờ thông tin sản phẩm; Bước 4: xây dựng danh mục TTT CCĐ bổ sung; Bước 5: xây dựng danh mục TTT CCĐ qua khảo sát ý kiến hội đồng chuyên môn.

ii) Phân tích thực trạng TTT CCĐ tại một số bệnh viện lớn trên địa bàn Tỉnh Quảng Ninh

Thiết kế nghiên cứu: mô tả cắt ngang.

Các bước thực hiện: Bước 1: code hoá các thuốc trong danh mục tương tác thuốc $\mathrm{CC}$; Bước 2: code hoá các thuật toán truy vấn các cặp tương tác khi xuất hiện trong 1 đơn thuốc trên phần mềm Navicat; Bước 3: xuất kết quả các cặp tương tác thuốc phát hiện được.

Xử lý số liệu: quản lý dữ liệu bằng excel và phân tích số liệu bằng phần mềm SPSS 20.0.

\section{Kết quả nghiên cứu}

3.1. Danh muc các cặp TTT CCĐ dụa trên danh muc thuốc thầu tập trung tại Tỉnh Quảng Ninh và thuộc phạm vi thanh toán của Qũ̃ BHYT.

Danh mục TTT CCĐ được xây dựng sau 5 bước trong phương pháp nghiển cứu. Kết quả thu được trình bày ở Bảng 1 .

Các hoạt chất có mặt trong một số lượng đáng kể các cặp tương tác trong danh mục này là Linezolid (15 cặp - chiếm tỷ lệ 13\%), Fluconazol (14 cặp - 12\%), Ketorolac (11 cặp - 10\%), Metoclopramid (11 cặp - 10\%), Amiodaron (10 cặp - 9\%) và Itraconazol ( 9 cặp - 8\%), còn lại là những hoạt chất chiếm tỷ lệ dưới $4 \%$. 
Bảng 1. Danh mục 112 cặp tương tác thuốc chống chỉ định

\begin{tabular}{|c|c|c|c|c|c|c|c|c|}
\hline $\mathrm{TT}$ & Cặp tương tác $C$ & & $\mathrm{TT}$ & Cặp tương tác $\mathrm{C}$ & & $\mathrm{TT}$ & Cặp tương tá & \\
\hline 1 & Alfuzosin & Amisulprid & 39 & Bezafibrat & Simvastatin & 76 & Fluconazol & Granisetron \\
\hline 2 & Amiodaron & Colchicin & 40 & Amiodaron & Vincamin & 77 & Fluconazol & Haloperidol \\
\hline 3 & Amisulprid & Azithromycin & 41 & Aceclofenac & Ketorolac & 78 & Fluconazol & Itraconazol \\
\hline 4 & Amisulprid & Ciprofloxacin & 42 & Acenocoumarol & Tamoxifen & 79 & Fluconazol & Ivabradin \\
\hline 5 & Amisulprid & Domperidon & 43 & Alfuzosin & Fluconazol & 80 & Fluconazol & Ondansetron \\
\hline 6 & Amisulprid & Famotidin & 44 & Alfuzosin & Itraconazol & 81 & Fluconazol & Quetiapin \\
\hline 7 & Amisulprid & Galantamin & 45 & Amiodaron & Amisulprid & 82 & Fluconazol & Salmeterol \\
\hline 8 & Amisulprid & Goserelin Acetat & 46 & Amiodaron & Fluconazol & 83 & Fluconazol & Tamoxifen \\
\hline 9 & Amisulprid & Granisetron & 47 & Amiodaron & Amitriptylin & 84 & Gemfibrozil & Simvastatin \\
\hline 10 & Amisulprid & Ivabradin & 48 & Amisulprid & $\begin{array}{l}\text { Benserazid/ } \\
\text { Levodopa }\end{array}$ & 85 & Haloperidol & Metoclopramid \\
\hline 11 & Amisulprid & Levofloxacin & 49 & Amisulprid & Clozapin & 86 & Ibuprofen & Ketorolac \\
\hline 12 & Amisulprid & Metoclopramid & 50 & Amisulprid & Erythromycin & 87 & Irinotecan & Itraconazol \\
\hline 13 & Amisulprid & Metronidazol & 51 & Amisulprid & Fluconazol & 88 & Itraconazol & Ivabradin \\
\hline 14 & Amisulprid & Moxifloxacin & 52 & Amisulprid & Itraconazol & 89 & Itraconazol & Midazolam \\
\hline 15 & Amisulprid & Octreotide & 53 & Amisulprid & Levodopa & 90 & Itraconazol & Simvastatin \\
\hline 16 & Amisulprid & Olanzapin & 54 & Amisulprid & Tamoxifen & 91 & Ketorolac & Meloxicam \\
\hline 17 & Amisulprid & Ondansetron & 55 & Amitriptylin & Linezolid & 92 & Ketorolac & Naproxen \\
\hline 18 & Amisulprid & Papaverin & 56 & Amitriptylin & Metoclopramid & 93 & Ketorolac & Pentoxifyllin \\
\hline 19 & Amisulprid & $\begin{array}{l}\text { Quetiapin } \\
\text { Fumarat }\end{array}$ & 57 & $\begin{array}{l}\text { Benserazid/ } \\
\text { Levodopa }\end{array}$ & Linezolid & 94 & Ketorolac & Piroxicam \\
\hline 20 & Amisulprid & Risperidon & 58 & $\begin{array}{l}\text { Benserazid/ } \\
\text { Levodopa }\end{array}$ & Sulpirid & 95 & Ketorolac & Tenoxicam \\
\hline 21 & Amisulprid & $\begin{array}{l}\text { Triptorelin } \\
\text { Pamoat }\end{array}$ & 59 & Bezafibrat & Ciprofibrat & 96 & Levodopa & Linezolid \\
\hline 22 & Amisulprid & Venlafaxin & 60 & Carbamazepin & Linezolid & 97 & Levodopa & Sulpirid \\
\hline 23 & Amisulprid & Chlorpromazin & 61 & Carbidopa & Linezolid & 98 & Linezolid & Methyldopa \\
\hline 24 & Clozapin & Fluconazol & 62 & Ciprofibrat & Fenofibrat & 99 & Linezolid & Mirtazapin \\
\hline 25 & Diclofenac & $\begin{array}{l}\text { Ketorolac } \\
\text { Tromethamin }\end{array}$ & 63 & Ciprofibrat & Gemfibrozil & 100 & Linezolid & Phenylephrin \\
\hline 26 & Carbamazepin & Tenofovir & 64 & Clozapin & Metoclopramid & 101 & Linezolid & Sertralin \\
\hline 27 & Flurbiprofen & $\begin{array}{l}\text { Ketorolac } \\
\text { Tromethamin }\end{array}$ & 65 & Codein & Linezolid & 102 & Linezolid & Sumatriptan \\
\hline 28 & Phenobarbital & Tenofovir & 66 & Colchicin & Erythromycin & 103 & Linezolid & Venlafaxin \\
\hline 29 & Phenytoin & Tenofovir & 67 & Colchicin & Itraconazol & 104 & Linezolid & Tramadol \\
\hline
\end{tabular}




\begin{tabular}{|l|l|l|l|l|l|l|l|l|}
\hline 30 & Amiodaron & Haloperidol & 68 & Dobutamin & Linezolid & 105 & Metoclopramid & Olanzapin \\
\hline 31 & Amiodaron & Moxifloxacin & 69 & Domperidon & Fluconazol & 106 & Metoclopramid & Quetiapin \\
\hline 32 & Amiodaron & Sulfamethoxazol & 70 & Dopamin & Linezolid & 107 & Metoclopramid & Risperidon \\
\hline 33 & Amiodaron & Trimethoprim & 71 & Erythromycin & Fluconazol & 108 & Metoclopramid & Sertralin \\
\hline 34 & Amiodaron & Chlorpromazin & 72 & Erythromycin & Simvastatin & 109 & Metoclopramid & Sulpirid \\
\hline 35 & Atenolol & Diltiazem & 73 & Etodolac & Ketorolac & 110 & Metoclopramid & Venlafaxin \\
\hline 36 & Bezafibrat & Fluvastatin & 74 & Etoricoxib & Ketorolac & 111 & Metoclopramid & Chlorpromazin \\
\hline 37 & Bezafibrat & Pravastatin & 75 & Felodipin & Itraconazol & 112 & Tamoxifen & $\begin{array}{l}\text { Warfarin } \\
\text { Sodium }\end{array}$ \\
\hline 38 & Bezafibrat & Rosuvastatin & & & & & & \\
\hline
\end{tabular}

3.2. Phân tích thực trạng tuơng tác thuốc chống chi định tại một số bệnh viện lớn trên địa bàn tỉnh Quảng Ninh

\subsubsection{Tần suất phát hiện cặp tuoong tác thuốc} chống chi định

Khảo sát 112 cặp TTT CCĐ trên dữ liệu điện tử từ 3 bệnh viện Tỉnh Quảng Ninh, kết quả phát hiện được 26 cặp tương TTT CCĐ. Xem chi tiết ở Bảng 2.
Có 297 bệnh án được phát hiện có TTT CCĐ, chiếm tỷ lệ $0,06 \%$. Trong đó, có 5 cặp tương tác phổ biến nhất là Amiodaron Amitriptylin (53 bệnh án - 54 đơn thuốc), Amiodaron-Moxifloxacin (35 bệnh án - 124 đơn thuốc), Amiodaron-Colchicin (32 bênh án - 110 đơn thuốc), Levodopa -Sulpirid (31 bệnh án - 96 đơn thuốc), Amiodaron-Haloperidon (19 bệnh án -35 đơn thuốc).

Bảng 2 . Tần suất 26 cặp tương tác thuốc chống chỉ định trên dữ liệu bệnh án nghiên cứu

\begin{tabular}{|l|l|l|l|l|l|}
\hline \multirow{2}{*}{ TT } & \multirow{2}{*}{ Cặp tương tác } & \multicolumn{2}{l|}{$\begin{array}{l}\text { Bệnh án có tương tác } \\
(\mathrm{N}=519.500)\end{array}$} & \multicolumn{2}{l|}{$\begin{array}{l}\text { Đơn thuốc có tương tác } \\
(\mathrm{N}=1.254 .099)\end{array}$} \\
\cline { 3 - 6 } & & $\begin{array}{l}\text { Số lượng } \\
\text { BA }\end{array}$ & Tỷ lệ \% & $\begin{array}{l}\text { Số lượng } \\
\text { đơn }\end{array}$ & Tỷ lệ \% \\
\hline 26 cặp TTT CCĐ & 297 & $0.06 \%$ & 789 & $0.06 \%$ \\
\hline 1 & Amiodaron-Amitriptylin & 53 & $0.0102 \%$ & 54 & $0.0043 \%$ \\
\hline 2 & Amiodaron-Moxifloxacin & 35 & $0.0067 \%$ & 124 & $0.0099 \%$ \\
\hline 3 & Amiodaron - Colchicin & 32 & $0.0062 \%$ & 110 & $0.0088 \%$ \\
\hline 4 & Levodopa - Sulpirid & 31 & $0.0060 \%$ & 96 & $0.0077 \%$ \\
\hline 5 & Amiodaron-Haloperidon & 19 & $0.0037 \%$ & 35 & $0.0028 \%$ \\
\hline 6 & Fluconazol - Ivabradin & 17 & $0.0033 \%$ & 128 & $0.0102 \%$ \\
\hline 7 & Haloperidon-Metocloramid & 17 & $0.0033 \%$ & 22 & $0.0018 \%$ \\
\hline 8 & Diclofenac - Ketorolac & 16 & $0.0031 \%$ & 16 & $0.0013 \%$ \\
\hline 9 & Benserazid/ Levodopa -Sulpirid & 14 & $0.0027 \%$ & 29 & $0.0023 \%$ \\
\hline 10 & Ketorolac - Piroxicam & 14 & $0.0027 \%$ & 14 & $0.0011 \%$ \\
\hline 11 & Amiodaron - Fluconazol & 11 & $0.0021 \%$ & 68 & $0.0054 \%$ \\
\hline
\end{tabular}




\begin{tabular}{|l|l|l|l|l|l|}
12 & $\begin{array}{l}\text { Amiodaron - } \\
\text { Sulfamethoxazol/Trimethoprim }\end{array}$ & 6 & $0.0012 \%$ & 20 & $0.0016 \%$ \\
\hline 13 & Fluconazol -Haloperidol & 6 & $0.0012 \%$ & 7 & $0.0006 \%$ \\
\hline 14 & Colchicin - Itraconazol & 5 & $0.0010 \%$ & 17 & $0.0014 \%$ \\
\hline 15 & Amiodaron-Clopromazin & 4 & $0.0008 \%$ & 10 & $0.0008 \%$ \\
\hline 16 & Itraconazol -Ivabradin & 3 & $0.0006 \%$ & 8 & $0.0006 \%$ \\
\hline 17 & Dobutamin -Linezolid & 2 & $0.0004 \%$ & 4 & $0.0003 \%$ \\
\hline 18 & Fluconazol -Itraconazol & 2 & $0.0004 \%$ & 10 & $0.0008 \%$ \\
\hline 19 & Itraconazol-Simvastatin & 2 & $0.0004 \%$ & 2 & $0.0002 \%$ \\
\hline 20 & Phenobarbital-Tenofovir & 2 & $0.0004 \%$ & 2 & $0.0002 \%$ \\
\hline 21 & Amitriptylin-Linezolid & 1 & $0.0002 \%$ & 8 & $0.0006 \%$ \\
\hline 22 & Carbamazepin -Linezolid & 1 & $0.0002 \%$ & 1 & $0.0001 \%$ \\
\hline 23 & Carbamazepin-Tenofovir & 1 & $0.0002 \%$ & 1 & $0.0001 \%$ \\
\hline 24 & Dopamine -Linezolid & 1 & $0.0002 \%$ & 1 & $0.0001 \%$ \\
\hline 25 & Itraconazol -Midazolam & 1 & $0.0002 \%$ & 1 & $0.0001 \%$ \\
\hline 26 & Ketorolac -Meloxicam & 1 & $0.0002 \%$ & 1 & $0.0001 \%$ \\
\hline
\end{tabular}

Bảng 3. Đặc điểm của bệnh nhân có tương tác thuốc chống chỉ định

\begin{tabular}{|l|l|l|l|}
\hline $\begin{array}{l}\text { Đặc điểm Bệnh viện } \\
\text { bệnh nhân }\end{array}$ & $\begin{array}{l}\text { Bệnh viện } \mathrm{A} \\
(\mathrm{N}=177,824)\end{array}$ & $\begin{array}{l}\text { Bệnh viện B } \\
(\mathrm{N}=191,192)\end{array}$ & $\begin{array}{l}\text { Bệnh viện C } \\
(\mathrm{N}=150,484)\end{array}$ \\
\hline Độ tuổi trung bình & 72,4 & 74 & 67 \\
\hline Giới tính (tỷ lệ nam/nữ) & $92 / 56$ & $3 / 1$ & $73 / 72$ \\
\hline Số bệnh án Nội trú & 132 & 3 & 137 \\
\hline Số bệnh án Ngoại trú & 16 & 1 & 8 \\
\hline
\end{tabular}

\subsection{2. Đặc điểm của bệnh nhân có tương tác thuốc chống chỉ định}

Trong số 297 bệnh án có phát hiện TTT $\mathrm{CCĐ}$, có nhiều bệnh án của cùng 1 bệnh nhân nhưng ở các đợt điều trị khác nhau. Kết quả được trình bày chi tiết trong Bảng 3 .

Độ tuổi trung bình của các bệnh nhân có TTT CCĐ ở 3 bệnh viện trên 67 tuổi. Trong số các bệnh án có TTT CCĐ, ở cả 3 bệnh viện, số lượng bệnh án nội trú cao hơn hẳn số bệnh án ngoại trú.
3.2.3. Tần suất phát hiện cặp tuoong tác thuốc CCĐ theo bệnh viện

Tần suất phát hiện bệnh án có TTT CCĐ tương đương ở 2 bệnh viện $\mathrm{A}$ và bệnh viện $\mathrm{C}$, lần lượt là $0,08 \%$ và $0,1 \%$. Trong khi đó, tần suất phát hiện bệnh án có TTT CCĐ ở bệnh viện $\mathrm{B}$ thấp hơn $(0,02 \%)$.

\subsubsection{Tỷ lệ phát hiện cặp tương tác thuốc CCĐ theo khoa diều trị}

Các khoa điều trị có bệnh án tương tác và tỷ lệ bệnh án phát hiện TTT CCĐ ở mỗi khoa được thể hiện chi tiết trong biểu đồ và Hình 1 . 
Bảng 4. Tần suất phát hiện cặp TTT CCĐ theo bệnh viện

\begin{tabular}{|l|l|l|l|}
\hline Bệnh viện & $\begin{array}{l}\text { Số lượng bệnh án có } \\
\text { tương tác CCĐ }\end{array}$ & Tổng số bệnh án khảo sát & Tần suất \\
\hline Bệnh viện A & 148 & 177,824 & $0,08 \%$ \\
\hline Bệnh viện B & 4 & 191,192 & $0,02 \%$ \\
\hline Bệnh viện C & 145 & 150,484 & $0.10 \%$ \\
\hline
\end{tabular}

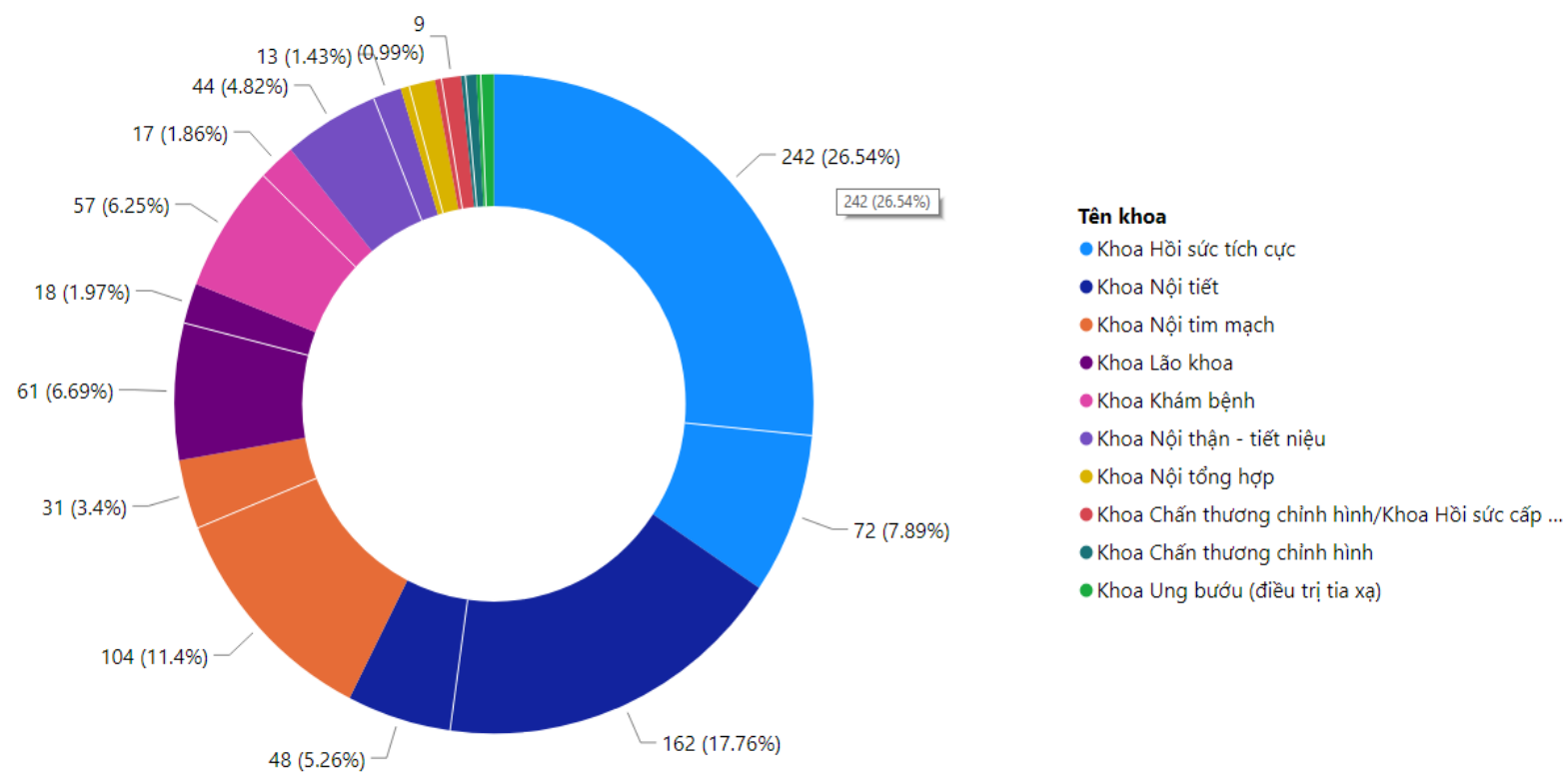

Hình 1. Tỷ lệ phát hiện cặp tương tác thuốc theo khoa điều trị.

Trong tổng số 297 bệnh án có TTT CCĐ, tỷ lệ phát hiện cao nhất tại các khoa Hồi sức cấp cứu $(24,2 \%)$, nội tiết $(16,2 \%)$, nội tim mạch $(10,4 \%)$, lão khoa $(6,1 \%)$. Tỷ lệ thấp hơn ở một số khoa thuộc khối Nội như Nội hô hấp $(5,1 \%)$, Nội thận-tiết niệu (4,4\%) và Nội tổng hợp (4\%). Số bệnh án có tương tác thuốc chống chỉ định thuộc các khoa, liên khoa còn lại có tỷ lệ dưới $2 \%$.

\section{Bàn luận}

Một trong những khó khăn khi xây dựng danh mục TTT cần chú ý trong thực hành lâm sàng là tính không tương đồng của các nguồn thông tin tra cứu, dẫn đến việc bỏ sót hoặc đưa ra "cảnh báo giả” [3]. Để khắc phục những khó khăn trên và thuận tiện tra cứu thông tin, đã có nhiều danh mục TTT bất lợi được xây dựng tại các cơ sở khám chữa bệnh [3], sau đó tích hợp vào phần mềm rà soát kê đơn điện tử. Trong nghiên cứu này nhóm tác giả tiến hành phân tích, rà soát TTT $\mathrm{CC}$ trên dữ liệu điện tử $\mathrm{BHYT}$ từ 3 bệnh viện Tỉnh Quảng Ninh thông qua phần mềm Navicat ${ }^{\circledR}$. Nghiên cứu được tiến hành qua hai giai đoạn: giai đoạn 1 - xây dựng danh mục TTT CCĐ lý thuyết, giai đoạn 2 - phân tích các TTT CCĐ thông qua phần mềm phân tích dữ liệu Navicat ${ }^{\circledR}$ có tích hợp các cặp TTT CCĐ lý thuyết. Giai đoạn 1 gồm 5 bước theo trình tự của nghiên cứu Nguyễn Thị Hạnh và cộng sự, 2019 [3]. Nhóm nghiên cứu sử dụng các phần mềm và tài liệu tra cứu bao gồm: Micromedex $2.0(\mathrm{MM}$ 2.0), tờ thông tin sản phẩm, tờ HDSD được ưu tiên tra cứu vì đây là $\mathrm{CSDL}$ được phê duyệt nội dung bởi Bộ Y tế Việt Nam [4]. Cuối cùng, tác 
giả tiến hành khảo sát ý kiến hội đồng chuyên môn để đưa ra danh mục TTT CCĐ hoàn thiện bao gồm 112 cặp TTT CCĐ lý thuyết trong đó thường gặp các hoạt chất như: Linezolid (15 cặp - chiếm tỷ lệ 13\%), Fluconazol (14 cặp - 12\%), Ketorolac (11 cặp - 10\%), Metoclopramid (11 cặp - 10\%), Amiodaron (10 cặp - 9\%) và Itraconazol (9 căp - 8\%). 112 TTT CCĐ là một con số khá chênh lệch khi so sánh với danh mục TTT CCĐ trong các nghiên trước như nghiên cứu trên danh mục thuốc của khoa khám bệnh cán bộ - Bệnh viện 108, tác giả Lê Thị Phương Thảo đưa ra 18 cặp TTT đồng thuận mức độ $\mathrm{CCĐ}$ giữa phần mềm $\mathrm{MM}$ và ít nhất $1 \mathrm{CSDL}$ khác, trong khi đó, trên danh mục thuốc bệnh viện Hợp Lực-Thanh Hóa, tác giả Lê Huy Dương chỉ báo cáo kết quả với 2 cặp TTT đồng thuận mức độ $\mathrm{CC}$ giữa $\mathrm{MM}$ và $\mathrm{DIF}[4,5]$. Sự chênh lệch đó là do danh mục thuốc nghiên cứu thuộc phạm vi đề tài là danh mục thuốc được áp dụng trên toàn Tỉnh Quảng Ninh, trong khi hầu hết các đề tài trên đều dựa trên danh mục 1 bệnh viện hay 1 khoa.

Trên cơ sở danh mục 112 TTT CCĐ lý thuyết, nhóm nghiên cứu phát hiện được 26 TTT CCĐ trên 789 đơn trong 297 bệnh án, chiếm tỷ lệ $0,06 \%$, trong đó có 272 bệnh án nội trú và 25 bệnh án ngoại trú. Trong nghiên cứu này TTT được phát hiện trên bệnh án đảm bảo 2 thuốc nằm trong cùng 1 đơn thuốc (cùng ngày y lệnh) và dựa vào danh mục TTT lý thuyết đã xây dựng, khác với nghiên cứu trước đó của các tác giả Lê Huy Dương và Tô Thị Hoài tìm ra cặp TTT có thể có trong bệnh án và không quy ước 2 thuốc đó nằm trong cùng 1 đơn thuốc $[4,6]$. Dựa trên tiêu chí số lượng bệnh án gặp TTT CCĐ, tần suất phát hiện cao nhất là amiodaron - amitriplin chiếm tỷ lệ $0,01 \%$ (53 bệnh án). Amiodaron là một một thuốc chống loạn nhịp tim nhóm III (theo phân loại Vaughan Williams), có tác dụng kéo dài khoảng QT và chuyển hóa qua CYP 450 [7]. Vì vậy, khi sử dụng đồng thời amiodaron với chất ức chế monoamin oxidase sẽ gây nguy cơ xoắn đỉnh, có thể ảnh hưởng nghiêm trọng thậm chí tử vong [1]. Hai cặp TTT CCĐ có tần suất phát hiện trên bệnh án cao thứ hai và thứ ba đều là các cặp liên quan đến amiodaron là cặp amiodaron - moxifloxacin và amiodaron cochicin với tỷ lệ lần lượt là $0,0067 \%$ (35 bệnh án) và $0,0062 \%$ (32 bệnh án).

Nghiên cứu được tiến hành trên 3 bệnh viện lớn tại tỉnh Quảng Ninh, việc khảo sát trên toàn bộ dữ liệu bệnh án điện tử của 3 bệnh viện này trong 1 năm cho phép bao quát hầu hết đặc điểm của quần thể bệnh nhân trên địa bàn tỉnh. Kết quả cho thấy không có sự khác biệt về số bệnh án chứa TTT $\mathrm{CC}$ của bệnh viện $\mathrm{C}$ (145 bệnh án) và bệnh viện $A$ (148 bệnh án) nhưng có sự khác biệt với bệnh viện $\mathrm{B}$ (4 bệnh án), có thể liên quan đến đặc điểm bệnh viện $\mathrm{B}$ là bệnh viện đã tích hợp phần mềm cảnh báo tương tác thuốc trên hệ thông kê đơn điện tử. Bệnh án có TTT CCĐ được phát hiện ở 13 khoa hoặc liên khoa khác nhau từ cả 3 bệnh viện, tỷ lệ phát hiện cao nhất tại các khoa Hồi sức cấp cưu $(24,2 \%)$, Nội tiết $(16,2 \%)$, Nội tim mạch $(10,4 \%)$ và Lão khoa $(6,1 \%)$. Khoa Hồi sức cấp cứu là nơi điều trị tích cực các trường hợp cấp cứu, nguy kịch nên việc đưa ra quyết định điều trị của bác sĩ thường chỉ được phép trong thời gian rất ngắn do đó việc tra cứu TTT từ các tài liệu chuyên khảo khó thực hiện được. Ngoài ra, trong nghiên cứu của Lê Thị Phương tại Bệnh viện Lão khoa Trung uơng, các khoa Can thiệp tim mạch, Nội tiết cũng là các khoa có tỷ lệ bệnh án có TTT cao nhất (xếp thứ tự lần lượt là thứ nhất - tỷ lệ cao nhất và thứ tư) [5].

Bệnh nhân gặp phải TTT CCĐ nằm trong độ tuổi tuổi từ 20 đến hơn 70 tuổi, trong đó chiếm tỷ lệ gặp phải TTT cao nhất là nhóm bệnh nhân trên 60 tuổi. Lý do được đưa ra là nhóm bệnh nhân này thường có nhiều bệnh mắc kèm do đó có thể sẽ sử dụng nhiều thuốc cùng lúc. So sánh với nghiên cứu của Heverton và cộng sự trên đối tượng bệnh nhân tiểu đường và mắc bệnh chuyển hóa, tỷ lệ gặp phải TTT tiềm tàng cũng cao nhất ở nhóm đồi tượng trên 60 tuổi $(79,5 \%)$ [8]. Tuy nhiên, hạn chế của nghiên cứu là chưa phân tích được tỷ lệ TTT theo số lượng thuốc trong đơn.

\section{Kết luận}

Nhóm nghiên cứu đã xây dựng danh mục 112 cặp TTT CCĐ dựa trên danh mục thuốc thầu tập trung tại Tỉnh Quảng Ninh và thuộc phạm vi 
thanh toán của Quỹ BHYT và đã phát hiện 297 bệnh án và 789 đơn có TTT CCĐ chiếm tỷ lệ $0,06 \%$, trong đó chủ yếu là đơn nội trú ở người cao tuồi.

\section{Tài liệu tham khảo}

[1] D.C. Malone, J. Abarca, P.D. Hansten, A.J. Grizzle, E.P. Armstrong, R.C.V Bergen, B.S. Duncan-Edgar, S.L. Solomon, R.B. Lipton, Indentification of Serious Drug-Drug Interactions: Results of the Partnership to Prevent Drug-Drug Interactions, J. Am. Pharm. Assoc. 44 (2004) 142151. https://doi.org/10.1331/154434504773062591.

[2] P. Vonbachl, A. Dubied, S. Kra“henbu“hl, J.H.Beer, Evaluation of frequently used drug interaction screening programs, Pharm. World. Sci. 30 (2008) 367-374.

https://doi.org/10.1007/s11096-008-9191-X.

[3] N.T. Hanh, V.T.P. Thao, H.Q. Tuan, N.X. Bach, N. T. Hai, Developing a list of important drugdrug interactions in the clinical practice in the internal department - Kien An Hospital, Hai Phong City, VNU Journal of Science: Medical and Pharmaceutical Sciences, 35(2) (2019) 54-67. https://doi.org/10.25073/2588-1132/vnumps.4179.
[4] L.H. Duong, Developing clinically signification drug-drug interactions at Hop Luc General Hospital (2017), Specialist Pharmacist Graduation thesis $-1^{\text {st }}$ Class, Ha Noi University of Pharmacy (in Vietnamese).

[5] L.T. Phuong, Managing clinically signification drug-drug interactions at the National Geriatric Hospital (2018), Pharmacist Graduation thesis, Ha Noi University of Pharmacy (in Vietnamese).

[6] T.T. Hoai, Examining drug-drug interactions in the inpatient medical records at the Thai Nguyen Lung Hospital (2017), Specialist Pharmacist Graduation thesis $-1^{\text {st }}$ Class, Ha Noi University of Pharmacy (in Vietnamese).

[7] Ministry of Health, Vietnam Regulatory Affairs Society, Medical Publishing House one member Company Limited, Vietnam, 2015 (in Vietnamese).

[8] A.P. Heverton, R.L.P Leonardo, M.V Carlos, F.F.C. Maria, Patient's lack of understanding producing insulin drug-interactions in Southeast Brazilian primary care clinics, Diabetes \& Metabolic Syndrome: Clinical Research \& Reviews. 3(2) (2019) 1131-1136. https://doi.org/10.1016/j.dsx.2019.01.032 